\title{
The sub-wavelength imaging performance of disordered wire media
}

\author{
David A. Powell \\ Nonlinear Physics Centre, Research School of Physical Sciences and Engineering, The Australian National University, Acton ACT, Australia
}

Received 15 February 2008; received in revised form 20 February 2008; accepted 20 February 2008

Available online 6 March 2008

Communicated by V.M. Agranovich

\begin{abstract}
An analysis of the sub-wavelength imaging performance of disordered thin wire media is undertaken, in order to understand how its performance may be affected by manufacturing errors. The structure is found to be extremely robust to disorder which keeps the wires parallel. Variation in the orientation of the wires and their longitudinal position causes more significant degradation in the image quality, which is quantified numerically. (C) 2008 Elsevier B.V. All rights reserved.
\end{abstract}

\section{Introduction}

The possibility of reconstructing an image with subwavelength details has been one of the driving forces behind the development of SRR/wire media composite metamaterials [1]. However, several studies [2-4] have shown that disorder suppresses the left-handed behavior, which is critical for this imaging. Robustness to disorder is essential when scaling a structure down to the scale of $\mathrm{THz}$ or optical wavelengths, where precise control of dimensions becomes more difficult.

An alternative structure for sub-wavelength imaging is the thin wire medium operating in the "canalisation regime" [5]. This structure consists of a square lattice of parallel wires oriented along the axis of the lens. This is a much simpler geometry which does not rely on resonant particles. It has also been shown that the structure can operate at higher frequencies where plasmonic effects become significant [6]. However its robustness to disorder has not been studied so far. In this Letter three different types of perturbation are made to the lens structure, and the resulting image quality is characterised.

\section{Method}

In order to investigate the sensitivity to error, a method of moments simulation of a finite-sized wire lens is performed,

E-mail address: david.a.powell@anu.edu.au. using a custom code for thin-wire structures with the basis functions described in [7]. This is a full wave model of the structure, with the simplification that the wires are considered to be perfect conductors, and to be thin in comparison to the wavelength. The lens structure considered is similar to that described in [5], with an operating frequency of $1 \mathrm{GHz}$, wires of length $\lambda / 2$ and radius $0.1 \mathrm{~mm}$ arranged in a $21 \times 21$ lattice with the pe$\operatorname{riod} a$ being $10 \mathrm{~mm}$. The wires are meshed into 30 sub sections each.

The image produced by the lens is calculated as the magnitude of the $z$-component of the electric field (i.e., the component parallel to the axis of the wires), within an area of size $20 \times 20 \mathrm{~cm}$, at a distance of $0.5 \mathrm{~mm}$ from the wire ends. As the imaging quality of such thin-wire lens structures is known to depend on the source spectrum [8], two different input spectra were used. The input images were generated by wire antennas placed $5 \mathrm{~mm}$ from the input end of the lens. Fig. 1 shows the output image for the two chosen input spectra. It can be seen that there are sharp field maxima at the locations of the wires. However, when considering imaging quality, details smaller than the lattice period should be ignored, since any practical detection system will employ some effective spatial averaging.

The corresponding output spectra are shown in Fig. 2. As these spectra are limited to $-\pi / a<k_{x}, k_{y}<\pi / a$, they do not contain the spurious information from the details smaller than the period. The changes in these spectra are used to quantify the 
effects of disorder. For each disordered lens the image error $E$ is defined as

$E=\frac{\int_{-\pi / a}^{\pi / a} \int_{-\pi / a}^{\pi / a}\left|I_{\mathrm{ref}}-I_{\mathrm{dis}}\right|^{2} d k_{x} d k_{y}}{\int_{-\pi / a}^{\pi / a} \int_{-\pi / a}^{\pi / a}\left|I_{\mathrm{ref}}\right|^{2} d k_{x} d k_{y}}$,

where $I_{\text {ref }}$ and $I_{\text {dis }}$ are the complex spectra of the reference and disordered lenses respectively.

We consider that the structure is designed to be regular and periodic, but suffers from some imperfections due to manufacturing defects. For a given level of disorder, it is desirable to estimate the error in a statistically meaningful way. It is most useful to construct an upper bounds on $E$, to find the worst case error which is likely to occur. The 95th percentile of $E$ was chosen to represent this upper bounds. This can be estimated using Eq. (9-25) from [9], and the estimate has been made with $95 \%$ confidence.

Fig. 3 shows the unit cell of the wire media, and illustrates the notation and reference directions used to describe the disorder of the wires.
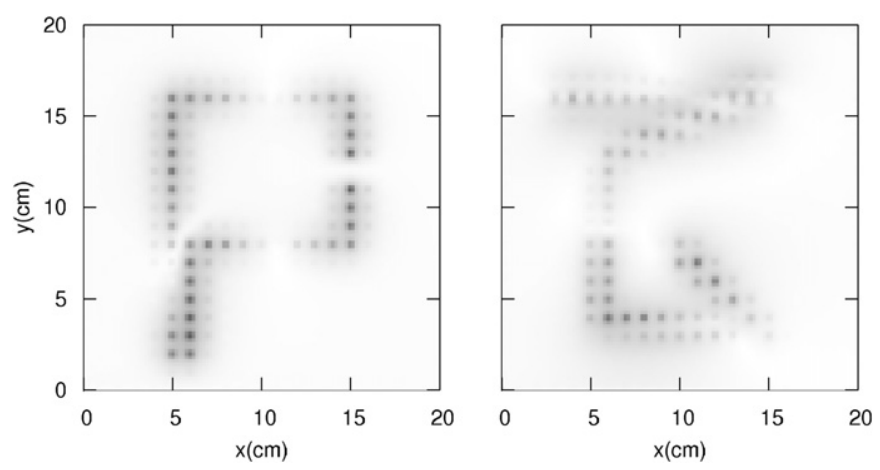

Fig. 1. Image reproduced at the output plane.
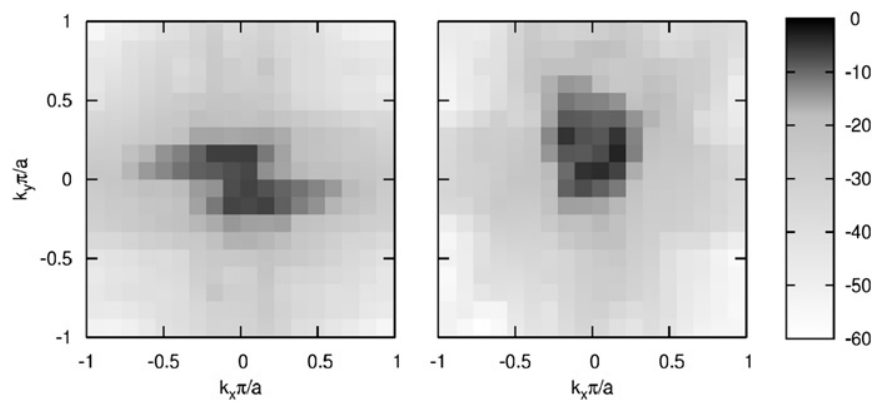

Fig. 2. Normalised magnitude of output spectra in dB.

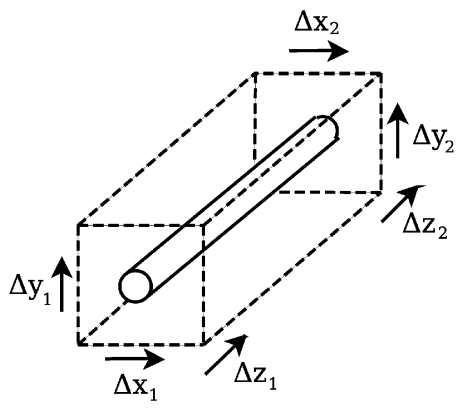

Fig. 3. Unit cell of wire media showing notation for perturbations.

\section{Results}

The first form of disorder considered was in the transverse position of the wires within their unit cells, with no change in their length or orientation, so that $\Delta x_{1}=\Delta x_{2}, \Delta y_{1}=\Delta y_{2}$, $\Delta z_{1}=\Delta z_{2}=0$. It seems physically reasonable to assume that the worst case error in the location of the wires would be to have them uniformly distributed within the unit cell.

Fig. 4 shows the resultant error distribution for both input spectra. The estimated upper bounds on the error due to uniformly distributed wire location was $3.8 \%$ for the first input spectrum, and $4.0 \%$ for the second. Given that these figures represent a worst case scenario for this type of disorder, it can be concluded that if the wires remain parallel, the image quality should similar to that of the regular structure.

The next form of disorder is with the wires being skew from parallel, but with the total length of the structure remaining fixed. This is achieved by having $\Delta x_{1}=-\Delta x_{2}, \Delta y_{1}=-\Delta y_{2}$, $\Delta z_{1}=\Delta z_{2}=0$. Small perturbations with Gaussian distribution were applied. Fig. 5 shows the resulting error as a function of $\sigma$, the standard deviation of $\Delta x$ and $\Delta y$. The number of samples was chosen such that the largest value of $E$ each $\sigma$ is the estimate of the 95 th percentile.

The final disorder applied to this structure was for the wires to be displaced slightly in the longitudinal direction by letting $\Delta x_{1}=\Delta x_{2}=0, \Delta y_{1}=\Delta y_{2}=0, \Delta z_{1}=\Delta z_{2}$. In this case both the source antenna and image plane remain at the same distance from the average position of the ends of the wires. The result-

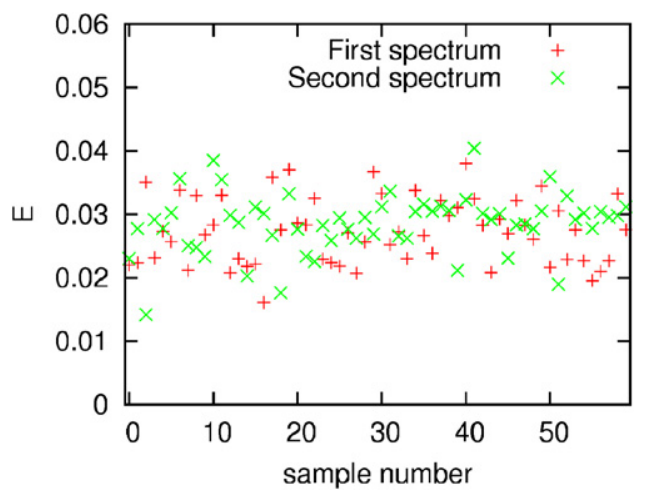

Fig. 4. Error due to uniform transverse displacement of wires.

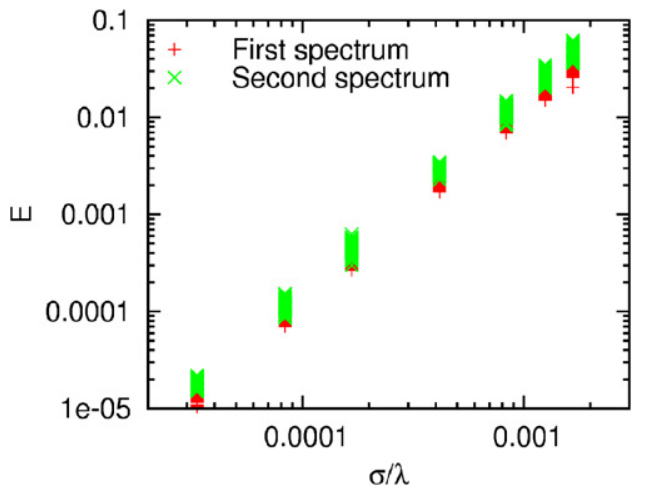

Fig. 5. Error due to skew of wires. 


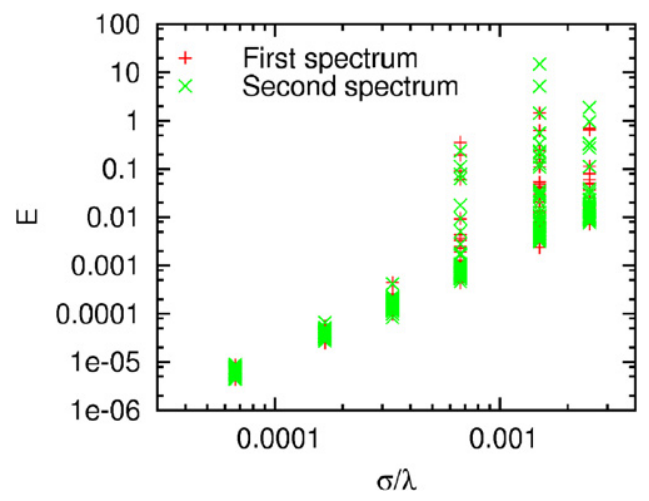

Fig. 6. Error due to longitudinal displacement of wires.

ing error is shown in Fig. 6, where $\sigma$ is the standard deviation of $\Delta z$, which has Gaussian distribution.

\section{Conclusion}

Examining Figs. 4, 5 and 6, it can be seen that the sensitivity to the three different types of disorder is quite different. If we consider that image degradation of around $10 \%$ is acceptable, then a very large degree of transverse displacement could be tolerated, but only a moderate amount of skew and a very minimal amount of longitudinal displacement would be acceptable.

This can be understood in terms of the uniaxial permittivity of the wire media [10], which is generally undisturbed by transverse displacement, but strongly distorted by skew. The displacement of wires along the length direction means that the wire medium is no longer a flat slab of length $\lambda / 2$, thus resulting in distortion due to impedance mismatch.

It should be noted that the error figures quoted here are relative to the unperturbed structure, which does not perfectly restore all spectral components, only transports TM polarised waves, and which may also influence the source field due to re- flections. The influence of disorder on the bandwidth of operation has not been considered. In addition the separation between the source and lens has been kept constant, and preliminary results indicate that this may be an important parameter in the sensitivity to disorder, most probably due to changes in reflection from the lens.

In conclusion, the sensitivity of wire media to disorder has been analysed. The results suggest that the wire media structure should be scalable to smaller dimensions as long as the wires can be kept close to parallel and their length is well controlled.

\section{Acknowledgements}

This work was supported by an award under the Merit Allocation Scheme on the APAC National Facility at the ANU. The author would like to thank Dr. Pavel Belov of Queen Mary University of London for useful discussions.

\section{References}

[1] J.B. Pendry, Phys. Rev. Lett. 85 (2000) 3966.

[2] J. Gollub, T. Hand, S. Sajuyigbe, S. Mendonca, S. Cummer, D.R. Smith, Appl. Phys. Lett. 91 (2007) 162907.

[3] M.V. Gorkunov, S.A. Gredeskul, I.V. Shadrivov, Y.S. Kivshar, Phys. Rev. E 73 (2006) 056605.

[4] K. Aydin, K. Guven, N. Katsarakis, C. Soukoulis, E. Ozbay, Opt. Express 12 (2004) 5896.

[5] P.A. Belov, Y. Hao, S. Sudhakaran, Phys. Rev. B 73 (2006) 033108.

[6] G. Shvets, S. Trendafilov, J.B. Pendry, A. Sarychev, Phys. Rev. Lett. 99 (2007) 053903.

[7] C. Chuang, J. Richmond, N. Wang, P. Pathak, IEEE Trans. Antennas Propagat. 38 (1990) 275.

[8] P.A. Belov, Y. Zhao, S. Sudhakaran, A. Alomainy, Y. Hao, Appl. Phys. Lett. 89 (2006) 262109.

[9] A. Papoulis, Probability, Random Variables, and Stochastic Processes, third ed., McGraw-Hill, 1991.

[10] P.A. Belov, R. Marqués, S.I. Maslovski, I.S. Nefedov, M. Silveirinha, C.R. Simovski, S.A. Tretyakov, Phys. Rev. B 67 (2003) 113103. 